\title{
Psycho-Traumatic Evaluation of Identity (PEI): Example of Depressive Disorder
}

\section{Joanic Masson ${ }^{1 *}$, Amal Bernoussi ${ }^{1}$, Yannick Gounden ${ }^{1}$, Charlemagne Simplice Moukouta1, François Erero Njiengwe ${ }^{2}$}

${ }^{1}$ Center of Psychology (EA 7273), University of Picardie Jules Verne, Amiens, France

${ }^{2}$ Laboratory of Bevavior Sciences, Université de Douala, Douala, Cameroun

Email: ‘joanic.masson@u-picardie.fr, amal.bernoussi@u-picardie.fr,yannick.gounden@u-picardie.fr, charlemagne.moukouta@u-picardie.fr,njiengwe@yahoo.com

How to cite this paper: Masson, J., Bernoussi, A., Gounden, Y., Moukouta, C.S. and Njiengwe, F.E. (2016) Psycho-Traumatic Evaluation of Identity (PEI): Example of Depressive Disorder. Open Journal of Psychiatry, 6, 262-272.

http://dx.doi.org/10.4236/ojpsych.2016.64031

Received: March 1, 2016

Accepted: October 21, 2016

Published: October 24, 2016

Copyright $\odot 2016$ by authors and Scientific Research Publishing Inc. This work is licensed under the Creative Commons Attribution International License (CC BY 4.0).

http://creativecommons.org/licenses/by/4.0/

\begin{abstract}
PEI is a clinical approach which aims at assessing the links between psycho-traumatic experience and depressive disorder. In the present study, inspired by the EMDR (Eye Movement Desensitization and Reprocessing) protocol, the PEI approach is proposed to 30 patients with depressive disorder. The aim is to determine the implication of negative cognitions and dysphoric emotions resulting from a traumatic experience on their psychopathological disorder. Our analysis shows a predominance of memories linked to a lack of affective support during childhood. The negative cognitions identified confirm a feeling of insecurity generated either by a failure of affective bond or helplessness towards the event. An alteration of self-esteem also emerges. Likewise, the present work also shows how psychotherapeutic care should consider narrowing the focus on traumatic experiences.
\end{abstract}

\section{Keywords}

PEI, EMDR, Psychological Trauma, Depressive Disorder, Psychotherapy

\section{Introduction}

The importance of psychological trauma in the etiology of psychopathology is well known to psychologists. However, few studies have focused specifically on the nature of the traumatic experience and its psychological and emotional consequences. More precisely, if we pay attention to the personnel history of patients, it is possible to observe tenuous links between the type of memories, the experiences of these and psychiatric disorders. The present research lies within this approach.

The use of PEI (Psycho-traumatic Evaluation of Identity) may be directed towards a 
twofold aim. It may be proposed as an aetiological evaluation of a psycho-pathological entity and is also of interest in choosing a potential psychotherapeutic care. It has emerged from the EMDR (Eye Movement Desensitization and Reprocessing) psychotherapy and our current knowledge of psychological trauma.

Following Shapiro's [1] first controlled study when it comes to understanding psychological traumas, psycho-pathologies and the therapeutic clinical approach (in particular, the development of Information Adaptive Treatment (IAT) model), the growing use of EMDR has broaden the perspectives. Initially proposed for the management of psycho-traumatic syndromes [2], an opening was made for a more diversified population with symptoms such as anxiety, inhibition, avoidance, alteration of self-esteem, depressive affect and algias. By proceeding with the anamnesis of these patients, an aetiology of their disorders and possible suitable care can be identified by using EMDR and free association processes achieved by bilateral stimulation.

On the basis of this literature, we propose in the present work to consider depressive disorders within the PEI clinical approach. The aim here is to bring out the important implication of psycho-traumatic aspects in the genesis of such type of pathology.

\section{Method}

EMDR was used with 210 patients within the framework of our psychotherapeutic practice. For each one of them, a "trauma mapping" was carried out. It consists in identifying memories which are painful, heavy, or numbing for the patient. These memories are then subject to desensitisation and reprocessing by eye movement and/or by alternating bilateral stimulation. The aim here is to identify what needs to be treated in accordance with the cognitive, emotional, behavioural and interpersonal consequences of the psychological traumas. This pragmatic approach has regularly revealed strong links which encompasses the traumatic life experience, the negative cognitions, identity construction and the psycho-pathological entity from which the patients suffer. Psychotraumatic Evaluation of Identity (PEI) is based on this analysis and has started to be worthy of interest in the framework of research in clinical and pathological psychology [3]-[5].

PEI consists of 4 steps:

Step 1 of PEI: to determine a list of ten painful memories and rank them in chrono$\underline{\text { logical order }}$

During a period of one to two weeks, the patient is asked to think upon and identify the ten most painful memories of his/her life. These memories must be sufficiently painful at the moment of the assessment by evoking sadness, anger, shame, guilt, or any other dysphoric feeling or sensation. They can be a part of the patient's life, a precise and short memory, or a recurring nightmare. It is not required to assess whether or not a given memory is linked to a real event. It can thus be a construction, a screen memory or a fantasy. The patient is requested to create a list which cannot exceed twelve items. If more memories are found, the patient is asked to choose the ten most painful ones. This way of processing is supported by clinical experience showing that these memories 
are most likely to reveal traumatic experiences. The patient is also requested to determine the approximate age of each event as much as possible and then rank the memories chronologically. If a memory triggers an emotion which is too painful to be put into words (shame, etc.), the patient may simply mention its existence, without broaching its content in order to avoid deepening the discomfort or suffering. Whilst this first exploratory step can be completed alone or with the psychologist, the rest of the PEI fits into a containing and reassuring clinical relationship in order to ease the oral expression of the memories and to avoid all risks of re-traumatisation. The clinician must be able to assess the patient's capacity to take on board his/her sufferings, in order to make the measures as therapeutic and suited as possible. In accordance with the modulation model of the neurophysiologic activation, the goal is to respect as much as possible the patient's window of tolerance [6] [7]. This can be perceived as a continuum going from under-activation (numbness of thought and affect) to emotional hyper reactivity. When the patient is far from these two extremes, his/her capacity to assimilate is preserved as well as his/her psychological balance.

Step 2 of PEI: to clear the image

The patient is invited to briefly recall the first memory. In this account, a sensory image usually relating to the most disturbing moment for the patient must be identified. In order to do so we ask the following question: "when you think about this memory, do images come to your mind? Sounds? Smells? Amongst all these images, these characteristics, which of them would be the most disturbing for you at this moment when recalling this memory?". The answers obtained are typically a clear picture proving the fixed and traumatic aspect of the memory [8].

Step 3 of PEI: to determine the negative cognition NC

Determining the most disturbing sensory image enables us to connect with the traumatic memory, and with a whole network of dysfunctional memories as well as their consequences on the psychological functioning of the patient. By focusing his/her attention on that image, the patient has to express what it makes him/her think of him/ herself in negative terms. The goal is to identify the consequences of that memory on the actual representation the patient has of himself/herself in his/her everyday life. In order to clarify this aspect, we formulate these types of questions: "when you think about this memory, particularly about the image (step 2) what does it make you think about yourself in negative terms and what do you still think today occasionally?". If a few NC arise, it is possible, depending on the expectations of the clinician, to determine the most significant one and to ask the patient which of the affirmations resonate in him/her the most today. A list of negative cognitions (annex 1), such as the one used in EMDR, can act as a medium for the patient to create his/her own list. Unlike the evaluative phase of the EMDR protocol where positive cognitions are sought, PEI only focuses on the psycho-traumatic impact of the experiences linked to the aetiology of the disorders.

Step 4 of PEI: to determine the emotions and sensations attached to the memory and the negative cognition 
The patient is asked to describe the emotions and feelings felt when focusing on both the initial image and the NC. The clinician thus asks the following questions: "When thinking about the starting image (step 2) and the sentence... (step 3), what emotions do you feel? What is happening in your body?". In this way the physiological emotions and feelings linked to that traumatic memory are brought up. We proceed in this manner for each of the patient's memories whilst respecting his/her capacity to create his/her story.

Synthetically, the four steps of PEI are summarised as follows:

Step 1 of the PEI: to determine a list of ten painful memories and rank them in chronological order.

Step 2 of the PEI: to define the image.

Step 3 of the PEI: to determine the negative cognition NC.

Step 4 of the PEI: to determine the emotions and sensations attached to the memory and the negative cognition.

Psycho-traumatic Evaluation of Identity generally allows to identify ten or so painful memories and to bring to light a dysfunctional aspect of the mnesic networks based on negative cognitions and emotions. The psycho-traumatic nature is, following EMDR, principally expressed by the sensory accuracy of the memory, the negative beliefs (NB) and the dysphoric emotions. Most anxious disorders studied within our clinical practice as well as a majority of depressive states, come from deleterious experiences that occurred in particular during childhood. These experiences implement a continuous system of emotions, cognitive distortions, behaviours and identity structures. The patients in question have built themselves based on their history and their representations of themselves and others underlined by emotions and behaviours which, by a feedback effect, become stronger and form the basis for building a dysfunctional identity.

We have applied PEI to 30 subjects with depression syndromes. Depressive states of any form are an essential public health problem which affects an increasing number of people. The risk factors such as: sex, age, socio-economical level, type of personality and life events, play a constant role in the variability of the depressive state. Loss of energy, sadness, psychomotor retardation and risks of suicide, are the most visible effects of depression affecting the professional, family, and affective life of the person. Resorting to care, hospitalisation and treatments (medication and classical psychotherapeutics) have given more or less beneficial results. If some depressions are linked to organicity, the role played by the individual's history, their way of dealing with conflicts, the socio-psychological factors strengthened by successive real or imaginary traumas are undeniable in the appearing, evolution and latency of the depressive state. Considering these elements helps in finding out the patient's unrevealed ill-being. PEI unveils this mask and contributes in identifying the consequences of some social, family, intra-psychological situations as well as the social values of an individualistic, pseudo competitive post-modern society, which may bring the person to a depressogenic collapse.

More precisely, our study focuses on the observations, medical records and data col- 
lections of patients seen in private practice. Data analysis was primarily clinical and qualitative. Patients were enrolled on the basis of the following principal criterion, the presence of a depressive syndrome with the given clinical signs: pathological sadness, psychomotor retardation, anxiety, and an alteration of self-esteem for at least three months. No psychotic patients took part in the present research.

\section{Results}

The mapping of psychological traumas of the population studied shows a dominance of traumatic memories which degrades self-esteem. In effect, we find in our clinical work: mentions of parents not investing themselves (parental conflicts, rejection, lack of interest, abuse) and memories linked to humiliation, loneliness and affective insecurity (aspects typical of abandonment anxiety, affective separations). We also highlight that for some of the patients, the death of a parent or a grandparent during childhood constitutes a particularly marking and painful event.

Negative cognitions unravelled (Table 1) show both an affective insecurity ("I am alone", "I have been abandoned") and a deep alteration of self-esteem ("I am not lovable", "I am worthless", "I am bad"). Cognitions linked to culpability ("it's my fault", "I am guilty") or to a coping issue ("I am powerless") can also be observed, thereby strengthening the narcissistic suffering. Emotions linked to these memories and cognitions are often sadness, shame, and guiltiness. Reminiscent of the depressive mood is also frequent. It is thus easy to understand the path followed by the consulting patients suffering from depressive disorders. Childhood is often marked by a lack of affection, which falls within an abandonment experience or rejection, humiliation and disappointment. Failures, separations and aggressions will during their lifetime, accentuate a retroflexion of aggressiveness towards themselves and favouring also a depreciation of the self. In effect, we often observe that aggressiveness constitutes the second emotional reaction towards a threat against the patient's physical and psychological integrity, after a reaction of fear which becomes by extension anguish or anxiety. This aggressiveness often takes the form of extreme choleric rage turned towards oneself, which degrades all the more the self-esteem, causing depressive suffering.

Table 1. Example of negative cognition in depressive disorder.

\begin{tabular}{cc}
\hline Theme of negative cognition & Example of negative cognition \\
\hline Affective insecurity & "I am alone" \\
"I have been abandoned" & "I am not lovable" \\
"I am worthless" & "I am bad" \\
Alteration of self-esteem & "It's my fault" \\
"I am guilty" & "I am powerless" \\
"I can't cope"
\end{tabular}


According to de O'Shea's work [9], trauma and negligence at an early age (0 to 3 years old), rarely accessible by language, are of particular importance. These memories which are archaic and inaccessible to semantic memory [8], may contain dysfunctional information that are generally linked: to a lack of emotional bond between baby and mother, to early health problems having affected the child's integrity, or to real abuse. The memories are rarely evoked during PEI, unless they have previously been mentioned within the family circle by a parent who was able to put them into words or inform the consultant. The hypothetical existence of this type of trauma must be considered when psychotherapies fail and the patient shows a dissociative symptomatology in the Janetien sense, that is a feeling of abandonment associated with emptiness which nothing can manage to fill or treat [10].

\section{Illustration of a Clinical Case}

Mrs. D, 45 years old, came to see us after having read a book on EMDR. Divorced, she brings up her 9 years old son alone. Her relationship with the dad is problematic (rarely present for about a year, history of psychological abuse). The patient describes an old malaise which originates in her childhood: strong alteration of self-esteem, recurring sadness and suicidal thoughts. Her sufferings brought her to be treated by many psychologists and psychiatrists in the past. Now under the care of a city psychiatrist, Mrs D. has been taking the same anti depressive treatment for the last 9 years. Both her parents died from a cancerous illness when she was 27 (mother) and 41 (father). The family context appears difficult, marked in particular by a longstanding anxio-depressive syndrome (mother) and an issue with alcohol (sister). It seems from the patient's discourse that many members of her family have a depressive pathology. Another important information is the patient's belief to be an undesired child. Indeed, abandonment elements are clearly present as well as elements of bulimia and hyporexia. During the first meeting we find typical signs of depressive syndrome: sadness, psychomotor retardation slightly masked by anxiety, deep alteration of self-esteem, suicidal thoughts without a clear scenario.

Here below is a list of traumatic events brought to light, as well as the corresponding negative cognitions and emotions. This list was developed with the psychologist:

1) Repeated absence of the mother for days (6/7 years old). NC: "I have been abandoned". Emotions: anguish, sadness.

2) Sudden death of the grandfather following a heart attack (8 years old). NC: "I am alone". Emotion: sadness, fear.

3) Lack of family nucleus-move (10 years old). NC: "I am insignificant". Emotion: sadness.

4) Alcohol and cannabis consumption as a teenager. NC: "I am worthless". Emotion: guilt, sadness, anguish.

5) Distancing from the mother as studying in another city (19 years old). NC: "I am alone". Emotion: sadness

6) Several romantic disappointments (19/25). NC: "I am weak". Emotion: sadness, 
anguish, shame, disgust.

7) Mother's cancerous illness and death (27). NC: "I have been abandoned". Emotion: sadness.

8) Pregnancy and absence of the spouse (35). NC: "I am alone". Emotion: sadness.

9) Death of the father (41). NC: "I am powerless". Emotion: sadness, anger.

10) Redundancy (42). NC: “I'm not worth anything”. Emotion: sadness.

11) Difficulty to stamp her authority on her son. NC: "I am a bad mother". Emotion: guilt, shame, anger, sadness.

Many of the memories brought to light in this clinical case relate to self-esteem. This can be seen through negative cognitions unveiled: "I am not worth anything", "I am rubbish", "I am a bad mother". These beliefs come from a life experience where the patient seems to fail or from memories lived with guilt, lessening once again a weakened self-esteem. Memories of loneliness can also be observed, bringing Mrs D to build a representation of herself around cognitions such as: "I have been abandoned", "I am alone", "I am insignificant". Elements typical of abandonment anxiety linked to a weak self-esteem, is a decisive factor in the alteration of the self (physical and psychological) and in the ability to both love and trust, and be loved. Classical painful emotions observed in numerous pathologies, in particular in anxio-depressive syndromes can be found in Mrs D's case: anxiety, fear, sadness, guilt, shame and anger. As seen earlier, depressive affects often come from a retroflexion phenomenon of aggressiveness, a mechanism which participates to self-disgust, pathological sadness and therefore to the alteration of self-esteem.

This aggressiveness constitutes an archaic reaction towards threat. In effect, ethology teaches us that in the face of adversity, the mammal often reacts with aggressiveness which can take two directions: fight or flight [11]. The same manifestations are observed in human beings. The aggressiveness often takes the form of an anger or rage turned towards oneself and less likely towards others. Not expressing the negative emotion is probably a means to limit the risk of accentuating the fear of rejection or abandonment by the others). This brings us to think that it is often a positive factor when the patient manages to feel this anger during psychotherapy. The aggressiveness is progressively reoriented towards the initial cause, where for example a parent was not able to play a sufficiently caring role. The patient is thus more into anxiety which constitutes a generalisation of fear, often of being abandoned or a fear of the one who was in the aggressor's position in the history of the patient. It is essential to go through this fear and assimilate these painful experiences.

\section{Discussion}

The dynamic analysis of traumas through PEI is a heuristic source which can guide us in our practice as clinicians and researchers in clinical psychopathologies and psychotherapies. This clinical evaluation sheds light on psycho-traumatic profiles linked to some pathologies. For example memories linked to loneliness, absence, lack of motherchild emotional bond, absence of the loved object can be found in most patients suffer- 
ing from an anxio-depressive syndrome. We also often identify experiences of aggressions, lack of benevolence, or experiences of failures which contribute in reinforcing the feeling of loneliness and the degradation of self-esteem. Events lived as traumatic during childhood, teenage years and/or adult life, bring the patient to build a pessimistic representation of the world as well as an alteration of the feeling of being able to face the world (feeling incomplete, helplessness linked to an alteration of self-esteem, the lack of adjustment strategies and psychomotor retardation). This can manifest itself both by cognitive distortions such as "I am useless" or "I am not lovable" and by adjustment difficulties towards everydays' stressors. Sometimes when a benign event resonates with earlier memories, it may trigger the first depressive decompression.

As a means of comparison with regard to patients with depressive disorders, we applied the same methodology to 20 patients with generalized anxiety disorder. The psychological trauma mapping on this population shows a predominance of memories linked to insecurity since childhood. Indeed, we find in our work memories linked to abuse and/or violence (parental conflicts, alcoholism, psychological violence, sexual abuse) and memories linked to illness, road accidents or death, and affective insecurity (elements typical of abandonment anxiety, affective separation). It is also worth mentioning that for some patients, financial insecurity of parents during childhood also constitutes a particularly marking or painful memory. Finally, the first panic attack is often experienced as a trauma which crystallises anxiety. The negative cognitions identified (Table 2) reveal a feeling of insecurity ("I am in danger", "I am going to die"), of helplessness towards the event ("I have no control over this", "I am weak") and an alteration of self-esteem often linked to an experience of abandonment ("I am rubbish", "I am alone", "I am abandoned", "I am not lovable"). The feeling linked to these memories and cognitions is most often fear, which, by generalisation, has turned into anxiety.

Through their history, in the course of their learning process, the patient builds a representation of him/herself which will shape his/her existence. This singularity will make him/her react in a certain way towards external situations (behavioural patterns). It is thus possible to observe during consultations links between psychological traumas, cognitive distortions, reassessment of identity and the type of relation with others. The notion of early inappropriate pattern developed by Young [12] helps in establishing

Table 2. Example of negative cognition in generalised anxiety disorder.

\begin{tabular}{cc}
\hline Theme of negative cognition & Example of negative cognition \\
\hline Insecurity & "I am in danger" \\
"I am going to die" & "I have no control over this" \\
"I am weak" & "I am rubbish" \\
& "I am alone" \\
"I am abandoned" & "I am not lovable" \\
\hline
\end{tabular}


these links.

\section{Implications for Theraputic Practice and Conclusion}

Psycho-traumatic Evaluation of Identity (PEI) from EMDR therapy can be applied to a diversified population or to specific disorders in order to better understand their aetiology and improve the psychotherapeutic care. The type of care considered here is an integrative therapy and plans to specifically target traumatic events. First we need to treat the 10 main traumatic events which seem to be the root of the anxio-depressive disorder. To do so, it is primordial to consider the ability of the patient to assimilate knowledge (tolerance window), to take into account the lack of psychological energy which can be treated with antidepressants and with the help of slow and progressive physical activities to bring back movement (meditation, qigong, walking, swimming, etc.).

A regular assessment of the suicidal intention must never be disregarded. It is essentially about treating the patient's past (painful memories brought to light using PEI), present (taking into account all of the contextual complexities which could participate in the suffering) as well as his/her future (help to project into the future) to anticipate any possible fear and generalise as much as possible the newly acquired resources. During the psychotherapeutic assessment, the negative beliefs tend to disappear allowing the creation of a new, positive representation of the self (positive cognitions). Dysphoric emotions lose their intensity until they finally disappear to make room for a stronger self-esteem and a more optimistic representation of the world with the development of new capacities to cope.

\section{References}

[1] Shapiro, F. (1989) Efficacy of the Eye Movement Desensitization Procedure in the Treatment of Traumatic Memories. Journal of Traumatic Stress, 2, 199-223. http://dx.doi.org/10.1002/jts.2490020207

[2] Bisson, J.L. (2007) Psychological Treatments for Chronic Post-Traumatic Stress Disorder. Systematic Review and Meta-Analysis. The British Journal of Psychiatry, 190, 97-104. http://dx.doi.org/10.1192/bjp.bp.106.021402

[3] Basset, L., Masson, J., Bernoussi, A. and Wawrzyniak, M. (2013) Cancer et terrain familial: Impact psychologique du risque de transmission d'un gène muté. Psycho-Oncologie, 7, 113-117. http://dx.doi.org/10.1007/s11839-013-0414-6

[4] Cozette Mience, M. (2012) Traumatisation, dissociation structurelle de la personnalité et addiction aux opiacés (Mémoire de master 2 de psychologie). Université de Picardie Jules Verne.

[5] Masson, J., Bernoussi, A., Cozette Mience, M. and Thomas, F. (2013) Complex Trauma and Borderline Personality Disorder. Open Journal of Psychiatry, 3, 403-407. http://dx.doi.org/10.4236/ojpsych.2013.34044

[6] Wilbarger, P. and Wilbarger, J. (1997) Sensory Defensiveness and Related Social/Emotional and Neurological Problems. Wilbarger, Van Nuys.

[7] Van der Kolk, B.A. (1987) Psychological Trauma. American Psychiatric Press, Washington DC. 
[8] Rothschild, B. (2008) Le corps se souvient: Mémoire somatique et traitement du trauma. De Boeck, Bruxelles.

[9] O'Shea, K. (2006) Reconstruction des fondations, reconnexion au Soi. Conférence EMDR, Istanbul.

[10] Grof, S. (1990) Psychologie Transpersonnelle. Editions du Rocher, Paris.

[11] Levine, P.A. (2010) In an Unspoken Voice: How the Body Releases Trauma and Restores Goodness. North Atlantic Books, New York.

[12] Young, J. (2005) La thérapie des schémas. Approche cognitive des troubles de la personnalité. De Boeck, Bruxelles.

[13] Shapiro, F. (2006) Manuel d'EMDR. Principes, Protocols, Procedures. Inter Editions, Paris. 
Annex 1: List of the Generic Negative and Positive Cognitions [13]

$\underline{\text { Responsibility/I am a bad person }}$

I do not deserve love.

I am a bad person.

I am horrible.

I am not worth anything.

I am not honourable.

I do not deserve to be loved.

I am not good enough.

I only deserve bad things.

I am never in good health.

I am not good-looking (my body is loathsome).

I do not deserve to...

I am stupid.

I am insignificant.

I am a catastrophe.

I deserve death.

I deserve to be unhappy.

I am not like others.

Responsibility/I have done something "wrong"

I should have done something.

I have done something wrong.

I should have known better.

Safety/vulnerability

I cannot be trusted.

I cannot (learn to) trust myself.

I cannot trust my judgement.

I cannot (learn to) protect myself.

I am in danger.

I cannot defend myself.

I am unarmed (defenceless).

Control/choice

I have no control.

I have no choice.

I am weak.

I cannot have what I want.

I always fail.

I must be perfect (be liked by everyone).

I cannot succeed.

I cannot bear it.

I am incompetent.

I cannot be trusted 